\title{
Expression of oncogenic long noncoding RNA PSMG3-antisense 1 in lung squamous cell carcinoma
}

\author{
E JIN ${ }^{1}$, CHAO HUANG ${ }^{1}$, LEI ZHANG $^{1}$, SHIYI CHEN $^{1}$, XIAOCHEN ZHAO $^{1}$, ZHENG REN $^{1}$ and HONG FU $^{2}$ \\ Departments of ${ }^{1}$ Medical Oncology and ${ }^{2}$ Interventional Oncology, \\ The Fourth People's Hospital of Shenyang, Shenyang, Liaoning 110031, P.R. China
}

Received January 27, 2021; Accepted July 23, 2021

DOI: $10.3892 / \mathrm{ol} .2021 .13012$

\begin{abstract}
Lung squamous cell carcinoma (LUSC) is one of the most common subtypes of lung cancer that accounts for $\sim 50 \%$ of all lung cancer cases. Long noncoding RNA (lncRNA) PSMG3-antisense (AS) 1 has been suggested to play an important role in various types of cancer. Therefore, the aim of the present study was to investigate the role of PSMG3-AS1 using clinical specimens and data from 130 patients with LUSC. The expression levels of PSMG3-AS1 and miR-143-3p were detected in LUSC specimens, and the correlation between lncRNA PSMG3-AS1 expression and patient clinical characteristics was analyzed. Cell Counting Kit-8, Transwell migration and invasion assays were used to investigate the functional role of PSMG3-AS1 in LUSC. The mechanism of PSMG3-AS1 on LUSC cells was also investigated using a luciferase activity assay with wild-type or mutated PSMG3-AS1. PSMG3-AS1 was found to be upregulated in LUSC, and high expression was associated with positive lymph node metastasis and a higher TNM stage. The results of multivariate Cox regression analysis revealed that PSMG3-AS1 may serve as an independent prognostic indicator in LUSC. Furthermore, inhibiting PSMG3-AS1 expression reduced tumor cell proliferative, migratory and invasive abilities. Moreover, PSMG3-AS1 was found to be closely associated with miR-143-3p in LUSC, and thus may become a potential prognostic marker and therapeutic target for the treatment of LUSC in the future.
\end{abstract}

\section{Introduction}

Lung cancer is one of the most common malignant tumors (1-3). Owing to the high incidence and mortality rates of lung cancer, it has become one of the most notable causes of

Correspondence to: Dr Hong Fu, Department of Interventional Oncology, The Fourth People's Hospital of Shenyang, 20 Huanghe Street, Shenyang, Liaoning 110031, P.R. China

E-mail: fu1224ting@163.com

Key words: invasion, long noncoding RNA PSMG3 antisense 1, lung squamous cell carcinoma, migration, microRNA-143-3p, prognosis, proliferation cancer-related death worldwide (4). According to pathological classification, lung cancer primarily includes small cell and non-small cell lung cancer (NSCLC) (5). NSCLC accounts for $\sim 66 \%$ of lung cancer cases $(6,7)$, among which lung squamous cell carcinoma (LUSC) is an important subtype (8). LUSC and its associated complications are reportedly responsible for $>400,000$ worldwide deaths annually (9). In addition to a lack of effective prognostic biomarkers, as LUSC frequently results in local infiltration and metastasis, the 5-year survival rate of patients with advanced disease is $<17 \%(10,11)$. Improving the prognosis of patients is likely to increase the survival rate $(12,13)$; therefore, it is necessary to identify reliable prognostic markers of LUSC for developing effective early therapeutic strategies.

Noncoding RNAs play an important regulatory role in the initiation of gene expression $(12,14,15)$. They are primarily divided into two subtypes, namely small noncoding RNAs [microRNAs (miRNAs/miRs)] with a transcript size of $<200$ nucleotides (nt), and long non-coding RNAs (lncRNAs) with a transcript size between $200 \mathrm{nt}$ and $100 \mathrm{~kb}$ (16-18). previous research on noncoding RNAs has mainly focused on miRNAs, and lncRNAs were often regarded as 'junk RNAs'. However, current literature demonstrates that the abnormal expression of lncRNAs directly influences the occurrence and development of different diseases, including various type of cancer $(13,17,19)$. Cui et al $(20)$ identified that PSMG3-antisense (AS) 1 serves as an oncogenic lncRNA in breast cancer, which has also been revealed to play an important role in lung adenocarcinoma (LUAD) (13). Investigating whether PSMG3-AS1 can associate with, or influence the biological functions of, cancer cells by interacting with miRNAs has become a major topic of interest. Numerous studies have suggested that a negative correlation exists between PSMG3-AS1 and miR-143-3p $(13,20)$. As reported, miR-143-3p targets PSMG3-AS1 in liver cancer, the expression levels of which were closely and inversely correlated (13); however, their association with LUSC remains to be elucidated. Therefore, the focus of the present study was to investigate the potential therapeutic significance of PSMG3-AS1 in LUSC.

\section{Materials and methods}

Patients and clinical specimens. A total of 130 patients, who were diagnosed with LUSC for the first time at The Forth 
People's Hospital (Shenyang, China) between Februry 2012 and December 2015, were selected. The inclusion criteria were: i) Diagnosed with LUSC by histopathological examination; ii) no history of tumor treatment; and iii) full clinical characteristics and 5-year follow-up information available. Paired LUSC and matched adjacent lung epithelial tissue specimens were collected during surgery, and confirmed by two pathologists. All specimens were stored in liquid nitrogen for further experimentation. The present study complied with the Ethics Committee of the Fourth People's Hospital of Shenyang [approval no. $\mathrm{k}(2012) 11$ ], and writtern informed consent was obtained from patients before the initiation of any study-related procedure. Patient clinicopathological characteristics were recorded (Table I), and information regarding 5 -year survival was obtained via telephone.

Cell lines and transfection. Human LUSC cell lines (H2170, H520, HCC95 and SK-MES-1) and the human lung epithelial BEAS-2B cell line were obtained from the Shanghai Cell Bank of the Chinese Academy of Sciences (Shanghai, China). All cells were cultured in DMEM supplemented with $10 \%$ FBS (both Gibco; Thermo Fisher Scientific, Inc.) at $37^{\circ} \mathrm{C}$ in a $5 \% \mathrm{CO}_{2}$ incubator.

Before transfection, LUSC cells $\left(2 \times 10^{5}\right.$ cells/well) were cultured in 6-well plates. Small interfering RNA (siRNA) targeting PSMG3-AS1 (si-PSMG3-AS1 sense, 5'-GGACGU CUCCCAUUCUGAATT-3' and antisense, 5'-UUCAGAAUG GGAGACGUCCTT-3') and the siRNA negative control (si-NC sense, 5'-UUCUCCGAACGUGUCACGUTT-3' and antisense, 5'-ACGUGACACGUUCGGAGAATT-3') were synthesized by Shanghai GenePharma Co., Ltd. The miR-143-3p inhibitor (5'-GAGCUACAGUGCUUCAUCUCA-3'), inhibitor NC (5'-CAGUACUUU UGUGUAGUACAA-3'), miR-143-3p mimic (5'-UGAGAUGAAGCACUGUAGCUC-3') and mimic NC (5'-UUCUCCGAACGUGUCACGUTT-3') were obtained from Guangzhou RiboBio Co., Ltd. All transfections were conducted at a final concentration of $50 \mathrm{nM}$ using Lipofectamine 3000 (Invitrogen; Thermo Fisher Scientific, Inc.) for $48 \mathrm{~h}$ at $37^{\circ} \mathrm{C}$ (according to the manufacturer's instructions), with untreated cells as the control. Follow-up experiments were carried out within $24 \mathrm{~h}$.

Reverse transcription-quantitative (RT-q) PCR. Total RNA was isolated from LUSC cells and tumor tissues using RNAzol (Sigma-Aldrich; Merck KGaA). To harvest miRNAs, RNA precipitation and washing were performed using $85 \%$ ethanol, with each centrifugation step at $13,840 \times \mathrm{g}\left(4^{\circ} \mathrm{C}\right)$ for $10 \mathrm{~min}$. A Precision nanoScript2 Reverse Transcription Kit (Primerdesign Ltd.) and miRNA 1st Strand cDNA Synthesis Kit (Vazyme Biotech Co., Ltd.) were used for reverse transcription of IncRNAs and miRNAs, respectively (A260/A280 ratio, 1.8-2.0). Subsequently, qPCR was conducted using a 7500 Real-Time PCR System (Applied Biosystems; Thermo Fisher Scientific, Inc.) with ChamQ SYBR qPCR Green Master Mix (Vazyme Biotech Co., Ltd.) and miRNA Universal SYBR qPCR Master Mix (Vazyme Biotech Co., Ltd.). The qPCR condition were as follows: $95^{\circ} \mathrm{C}$ for $30 \mathrm{sec}$, followed by 40 cycles at $95^{\circ} \mathrm{C}$ for $15 \mathrm{sec}, 60^{\circ} \mathrm{C}$ for $30 \mathrm{sec}, 72^{\circ} \mathrm{C}$ for $15 \mathrm{sec}$, and then final extension at $72^{\circ} \mathrm{C}$ for $5 \mathrm{~min}$. GAPDH and U6 were used as the endogenous controls for PSMG3-AS1 and miR-143-3p, respectively. The expression levels of PSMG3-AS1 and miR-143-3p were calculated using the $2^{-\triangle \Delta C q}$ method (21), and were normalized to those of GAPDH and U6, respectively. The primer sequences were as follows: PSMG3-AS1 forward, 5'-AAATGTGGGAGG GATGGCAG-3' and reverse, 5'-AATGGTGCCTTCCCCATC AG-3'; GAPDH forward, 5'-CTGGGCTACACTGAGCAC C-3' and reverse, 5'-AAGTGGTCGTTGAGGGCAATG-3'; miR-143-3p forward, 5'-CTGGCGTTGAGATGAAGCAC-3' and reverse, 5'-CAGAGCAGGGTCCGAGGTA-3'; and U6 forward, 5'-CGCTTCGGCAGCACATATAC-3' and reverse, 5'-TTCACGAATTTGCGTGTCATC-3'.

Cell Counting Kit 8 (CCK-8) analysis. The CCK-8 kit (Dojindo Molecular Technologies, Inc.) was used to assess H520 and SK-MES-1 cell proliferative capacity. Transfected cells were seeded into 96 -well culture plates $\left(4 \times 10^{3}\right.$ cells/well) and cultured in a humidified incubator with $5 \% \mathrm{CO}_{2}$ at $37^{\circ} \mathrm{C}$ for $0,24,48$ and $72 \mathrm{~h}$. Subsequently, $10 \mu \mathrm{l} \mathrm{CCK}-8$ solution was added. After a further 2-h incubation, the absorbance value of each well was measured at $450 \mathrm{~nm}$ using a microplate reader (Thermo Fisher Scientific, Inc.).

Transwell migration and invasion assays. A 24-well Transwell plate $(8 \mu \mathrm{m}$ pore; Corning, Inc.) was used for Transwell migration and invasion assays. The experimental procedure was similar in both the migration and invasion assays, except that the upper chamber was precoated with Matrigel (BD Biosciences) for $6 \mathrm{~h}$ at $37^{\circ} \mathrm{C}$ in the invasion assay. Briefly, transfected cells $\left(5 \times 10^{4}\right.$ cells/well) were seeded into the upper chamber in serum-free DMEM medium, and DMEM with $10 \%$ FBS was added to the lower chamber as a chemoattractant. After incubation for $24 \mathrm{~h}$ at $37^{\circ} \mathrm{C}$, the migrated or invaded cells were fixed with $4 \%$ paraformaldehyde for $15 \mathrm{~min}$ at room temperature, and stained with $0.1 \%$ crystal violet for $20 \mathrm{~min}$ at room temperature. The number of cells was observed under a light microscope in five random fields of view.

Bioinformatisc analysis. The interaction between PSMG-AS1 and miR-143-3p was predicted using starBase v2.0 database (http://starbase.sysu.edu.cn/index.php).

Dual-luciferase reporter assay. The PSMG3-AS1 and corresponding mutant sequences (without PSMG3-AS1 binding sites) were synthesized and subcloned into luciferase reporter vectors (Promega Corporation), which were subsequently named WT-PSMG3-AS1 and MUT-PSMG3-AS1, respectively. SK-MES-1 cells were then seeded into 24-well plates and co-transfected with miR-143-3p mimics, miR-143-3p inhibitor, mimic-NC and inhibitor-NC (as aforementioned) using Lipofectamine ${ }^{\circledR} 2000$ (Invitrogen; Thermo Fisher Scientific, Inc.). After $48 \mathrm{~h}$, the firefly luciferase activity was determined using a dual-luciferase reporter assay kit (Promega Corporation) in accordance with the manufacturer's protocol and normalized to Renilla luciferase activity.

Statistical analysis. Statistical analysis was performed using SPSS 20.0 (IBM Corp) and GraphPad Prism 5.0 software (GraphPad Software, Inc.). All experiments were repeated at least three times, and the data are presented as the 
Table I. Correlation between PSMG3-AS1 expression and the clinical characteristics of patients with lung squamous cell carcinoma.

PSMG3-AS1 expression

\begin{tabular}{|c|c|c|c|c|}
\hline & & & & \\
\hline Characteristic & Cases (n130) & Low $(n=60)$ & High $(n=70)$ & P-value \\
\hline Age & & & & 0.663 \\
\hline$\leq 60$ & 58 & 28 & 30 & \\
\hline$>60$ & 72 & 32 & 40 & \\
\hline Sex & & & & 0.828 \\
\hline Male & 68 & 32 & 36 & \\
\hline Female & 62 & 28 & 34 & \\
\hline Tumor size & & & & 0.745 \\
\hline$\leq 5 \mathrm{~cm}$ & 67 & 30 & 37 & \\
\hline$>5 \mathrm{~cm}$ & 63 & 30 & 33 & \\
\hline Smoking status & & & & \\
\hline Non-smoker & 65 & 34 & 31 & 0.159 \\
\hline Smoker & 65 & 26 & 39 & \\
\hline Differentiation & & & & 0.172 \\
\hline Well, Moderate & 74 & 38 & 36 & \\
\hline Poor & 56 & 22 & 34 & \\
\hline Lymph node metastasis & & & & 0.001 \\
\hline Negative & 80 & 46 & 34 & \\
\hline Positive & 50 & 14 & 36 & \\
\hline TNM stage & & & & 0.006 \\
\hline I, II & 81 & 45 & 36 & \\
\hline III, IV & 49 & 15 & 34 & \\
\hline
\end{tabular}

PSMG3-AS1, long noncoding RNA PSMG3-antisense 1.

mean \pm standard deviation. The $\chi^{2}$ test was used to determine the association between PSMG3-AS1 expression and patient clinical data. One-way ANOVA followed by Tukey's post hoc test was used to determine significant differences among multiple groups, and paired Student's t-test was used for comparing data between two groups. Kaplan-Meier (and the log-rank test) and Cox regression analysis were used to evaluate the prognostic significance of PSMG3-AS1, and Pearson's test was used for correlation analysis. $\mathrm{P}<0.05$ was considered to indicate a statistically significant difference.

\section{Results}

Expression of PSMG3-AS1 in LUSC tissues and cell lines. PSMG3-AS1 expression levels in LUSC and adjacent-normal tissues were determined using RT-qPCR analysis. Compared with adjacent-normal tissues, the expression levels of PSMG3-AS1 were higher in tumor tissues (Fig. 1A; $\mathrm{P}<0.001$ ). In addition, the expression of PSMG3-AS1 in four different LUSC cell lines and one normal cell line was assessed. As illustrated in Fig. 1B, the expression of PSMG3-AS1 was higher in all LUSC cell lines than in the normal cell line (all $\mathrm{P}<0.05$ ). The two cell lines exhibiting the highest relative expression of PSMG3-AS1 (SK-MES-1 and H520) were selected for subsequent experimentation. These results indicated that PSMG3-AS1 may play an oncogenic role in LUSC.

Association between PSMG3-AS1 and clinicopathological characteristics of patients with LUSC. The association between PSMG3-AS1 expression and the clinical parameters of patients with LUSC was evaluated to determine whether PSMG3-AS1 expression was involved in LUSC tumor progression. Patients were divided into low (60 patients) and high (70 patients) PSMG3-AS1 expression groups, based on the mean PSMG3-AS1 expression value. The $\chi^{2}$ test revealed that upregulated PSMG3-AS1 expression was associated with TNM stage $(\mathrm{P}=0.006)$ and lymph node metastasis $(\mathrm{P}=0.001)$, suggesting that PSMG3-AS1 expression may be involved in the progression of LUSC. However, PSMG3-AS1 expression was not significantly associated with any of the other investigated characteristics, including age, sex, tumor size, smoking status, and tumor differentiation $(\mathrm{P}>0.05$; Table I).

Association between PSMG3-AS1 and the prognosis of LUSC patients. Kaplan-Meier curve analysis and the log-rank test were used to assess the prognostic value of PSMG3-AS1 in LUSC. Based on the PSMG3-AS1 expression and overall survival status of patients with LUSC, the results demonstrated 

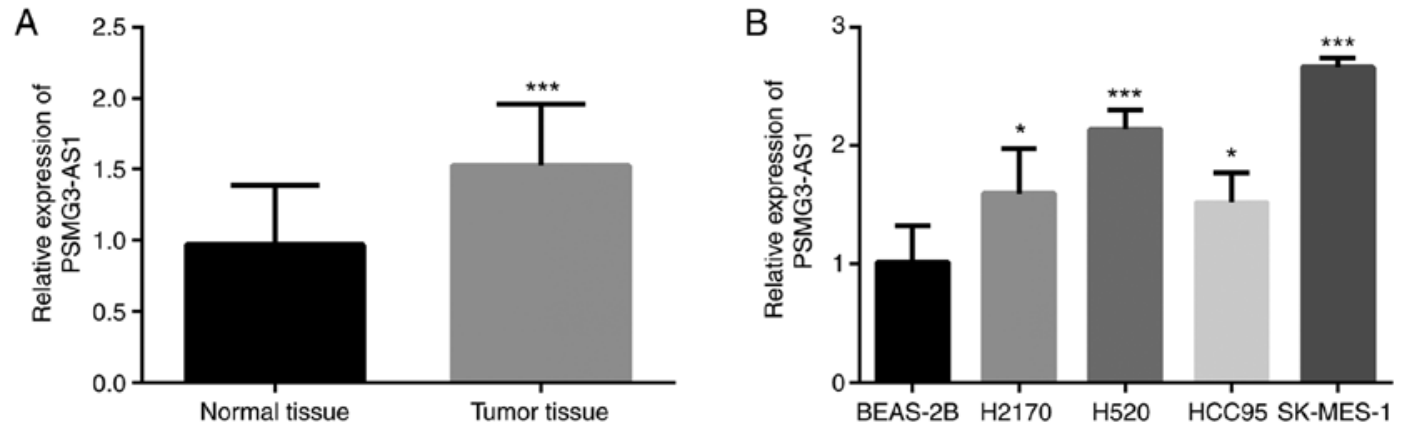

Figure 1. PSMG3-AS1 is upregulated in LUSC tissue samples and cells. (A) Relative expression of PSMG3-AS1 in LUSC and corresponding normal tissue samples. (B) Expression of PSMG3-AS1 in four LUSC cell lines and a normal epithelial cell line, BEAS-2B. " $\mathrm{P}<0.05$ and ${ }^{* * * *} \mathrm{P}<0.001$ vs. normal tissue or BEAS-2B cells. PSMG3-AS1, long noncoding RNA PSMG3-antisense 1; LUSC, lung squamous cell carcinoma.

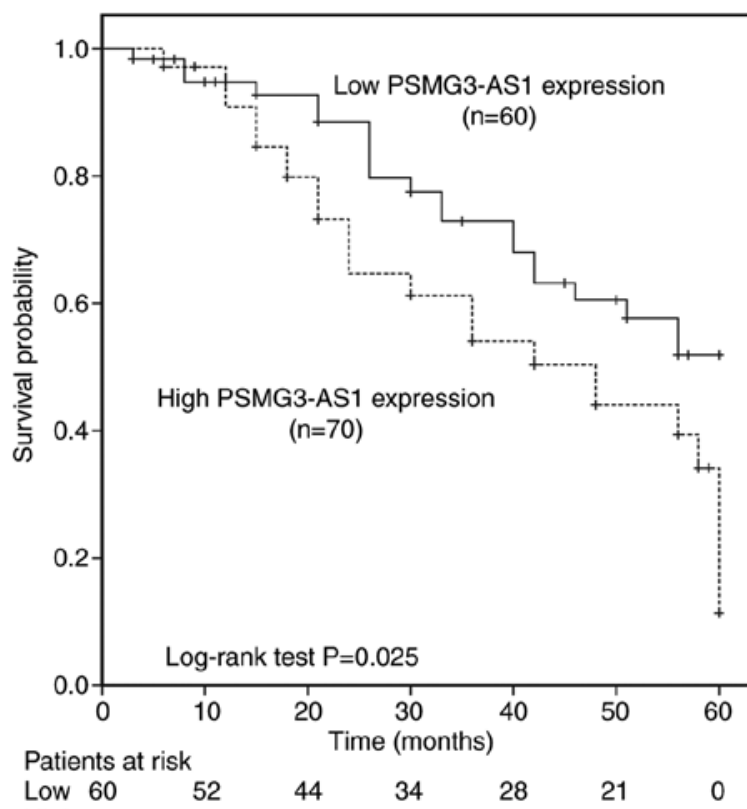

$\begin{array}{lllllll}\text { High } 70 & 62 & 44 & 34 & 28 & 21 & 0 \\ & 62 & 48 & 34 & 29 & 19 & 0\end{array}$

Figure 2. Kaplan-Meier analysis of the 5-year survival rate of LUSC patients with high and low expression levels of PSMG3-AS1. Patients with high PSMG3-AS1 expression exhibited a lower overall survival rate than those with low PSMG3-AS1 expression. PSMG3-AS1, long noncoding RNA PSMG3-antisense 1; LUSC, lung squamous cell carcinoma.

that the 5-year overall survival rate of patients in the high PSMG3-AS1 expression group was lower than that of those in the low expression group ( $\mathrm{P}=0.025$; Fig. 2). Moreover, multivariate Cox regression analysis indicated that PSMG3-AS1 expression level (hazard ratio, 2.068; $95 \%$ confidence interval, 1.142-3.744; $\mathrm{P}=0.016$ ) was an independent prognostic factor for assessing the 5-year overall survival of patients with LUSC (Table II). These results suggested that PSMG3-AS1 may be a prognostic marker for LUSC.

PSMG3-AS1 promotes LUSC cellular characteristics. To determine its functional role in LUSC, PSMG3-AS1 expression in H520 and SK-MES-1 cells was quantified using RT-qPCR following si-PSMG3-AS1 transfection. As illustrated in Fig. 3A and B, PSMG3-AS1 expression was significantly decreased by si-PSMG3-AS1 in H520 and SK-MES-1 cells $(\mathrm{P}<0.01)$. Subsequently, a CCK-8 assay was performed to
Table II. Multivariate Cox analysis of the clinical characteristics of patients with lung squamous cell carcinoma in relation to overall survival.

\begin{tabular}{lccc}
\hline & \multicolumn{3}{c}{ Multivariate analysis } \\
\cline { 2 - 4 } Characteristic & HR & $95 \%$ CI & P-value \\
\hline PSMG3-AS1 & 2.068 & $1.142-3.744$ & 0.016 \\
Age & 0.784 & $0.467-1.317$ & 0.358 \\
Sex & 0.908 & $0.535-1.540$ & 0.720 \\
Tumor size & 1.127 & $0.669-1.898$ & 0.654 \\
Smoking status & 1.477 & $0.878-2.486$ & 0.142 \\
Differentiation & 1.466 & $0.863-2.490$ & 0.157 \\
Lymph node metastasis & 1.728 & $1.004-2.975$ & 0.048 \\
TNM stage & 1.730 & $1.014-2.953$ & 0.044 \\
\hline
\end{tabular}

PSMG3-AS1, long noncoding RNA PSMG3-antisense 1.

assess cellular proliferative capacity. The results indicated that PSMG3-AS1-knockdown suppressed cellular proliferation $(\mathrm{P}<0.001$; Fig. 3C and D). Moreover, the Transwell assay results demonstrated that si-PSMG3-AS1 suppressed the migratory and invasive abilities of H520 and SK-MES-1 cells $(\mathrm{P}<0.01$; Fig. 3E-H). Of note, no marked differences were observed between the H520 and SK-MES-1 cell lines in characterizing LUSC. Ultimately, these findings confirmed the oncogenic role of PSMG3-AS1 in LUSC cells.

Interaction between PSMG3-AS1 and miR-143-3p. The SK-MES-1 cell line (in which the expression of PSMG3-AS1 was the highest) was used to determine the association between miR-143-3p and PSMG3-AS1. According to the bioinformatics analysis, miR-143-3p and PSMG3-AS1 formed multiple base pairings (Fig. 4A). Spearman's rank correlation coefficient analysis was used to evaluate the correlation between PSMG3-AS1 and miR-143-3p. The findings indicated that the expression of PSMG3-AS1 was inversely correlated with that of miR-143-3p ( $\mathrm{r}=-0.8152 ; \mathrm{P}<0.0001$; Fig. 4B). The silencing of PSMG3-AS1 in SK-MES-1 cells was confirmed by RT-qPCR, and the results revealed that miR-143-3p expression was successfully increased $(\mathrm{P}<0.01$; 

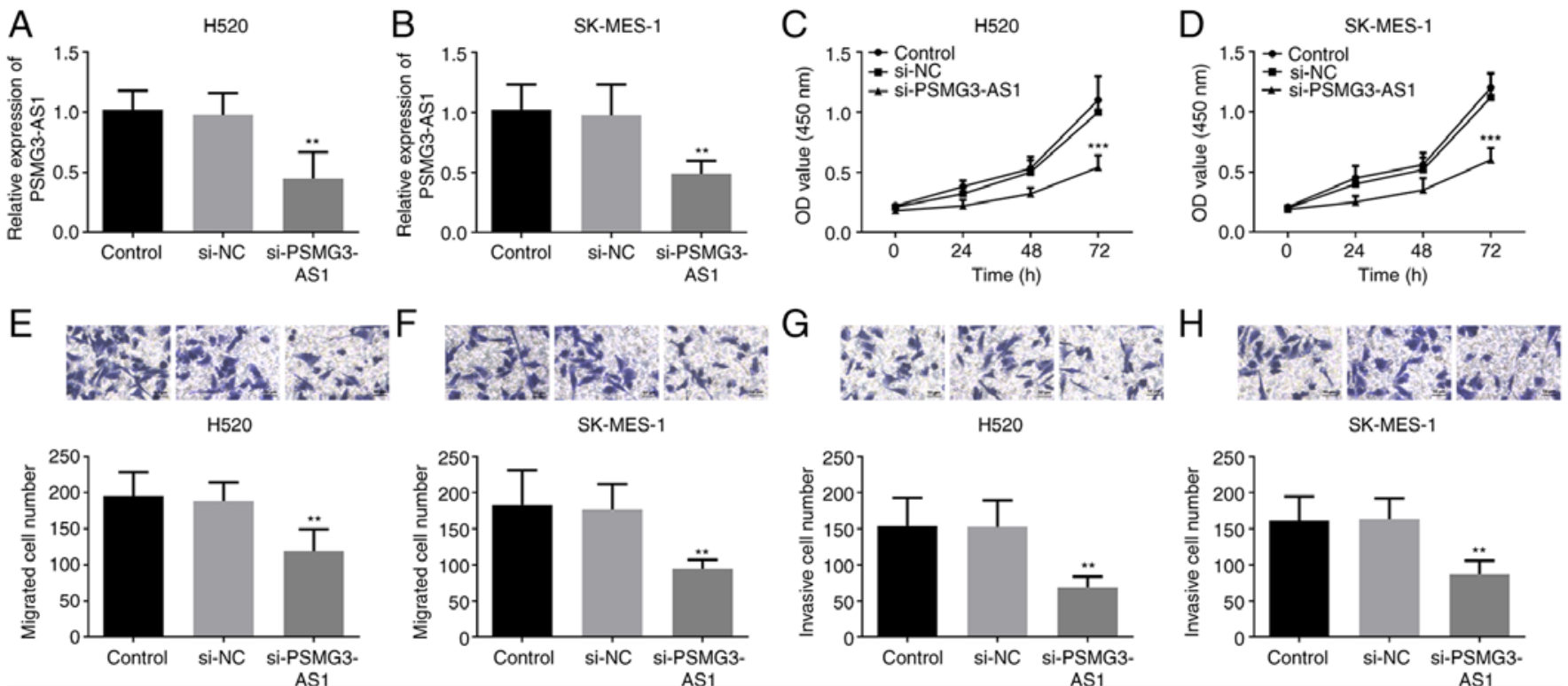

Figure 3. Proliferation of H520 and SK-MES-1 cells is suppressed following PSMG3-AS1-knockdown. H520 and SK-MES-1 cells were transfected with si-NC or si-PSMG3-AS1. Expression level of PSMG3-AS1 was determined in (A) H520 and (B) SK-MES-1 cells using reverse transcription-quantitative PCR. Proliferative capacity of (C) H520 and (D) SK-MES-1 cells was determined by Cell Counting Kit 8 analysis. Migratory ability of (E) H520 and (F) SK-MES-1 cells was determined by Transwell assays. Invasive ability of (G) H520 and (H) SK-MES-1 cells was measured by Matrigel assays. Scale bar, $50 \mu \mathrm{m} ;$ Magnification, x200. ${ }^{* *} \mathrm{P}<0.01$ and ${ }^{* * *} \mathrm{P}<0.001$ vs. si-NC. PSMG3-AS1, long noncoding RNA PSMG3-antisense 1; si, small interfering; NC, negative control.
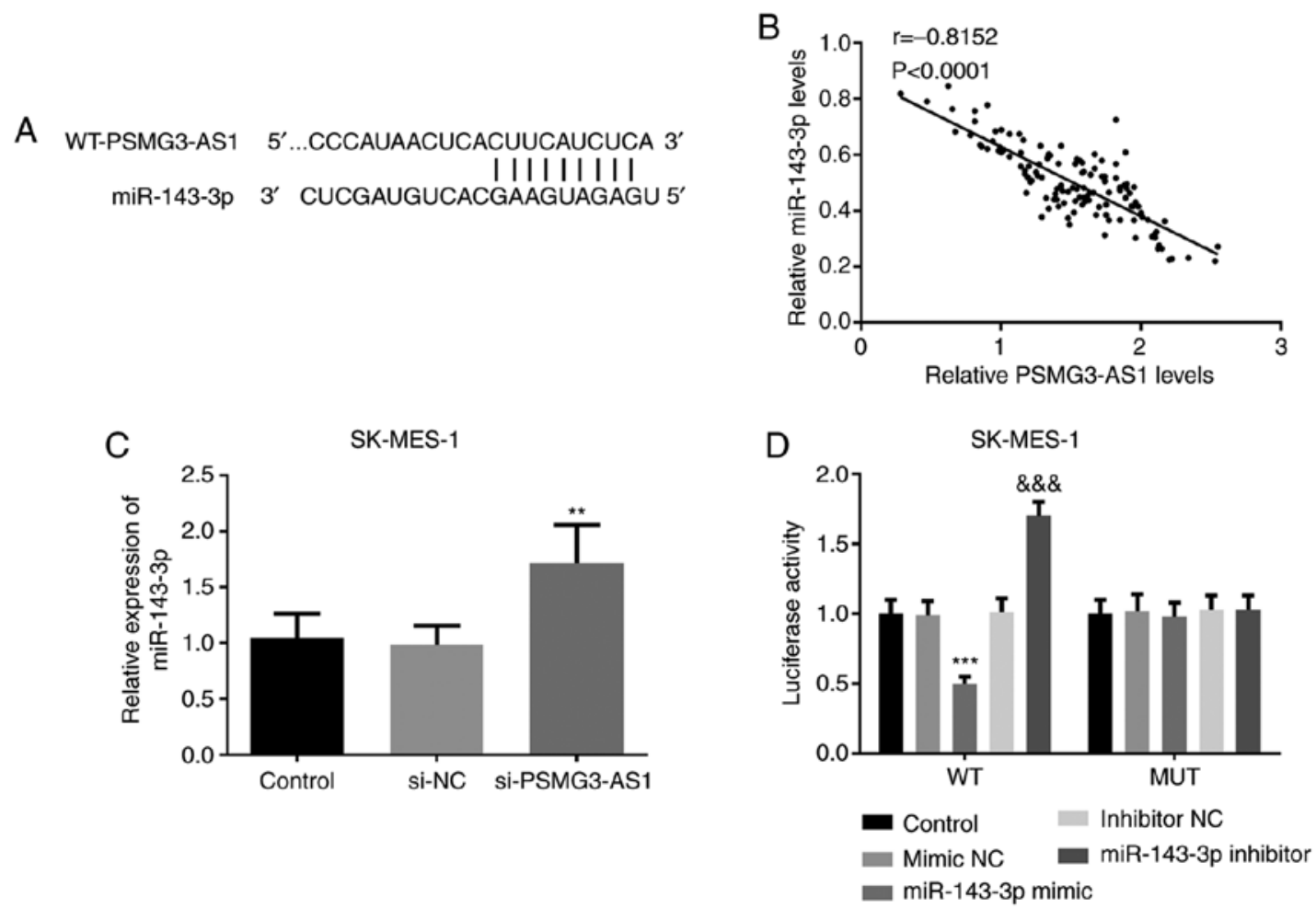

Figure 4. PSMG3-AS1 interacts with miR-143-3p. (A) Common binding site between miR-143-3p and PSMG3-AS1. (B) Correlation between miRNA-143-3p and PSMG3-AS1 expression in lung squamous cell carcinoma tissues was assessed using Pearson's correlation analysis. (C) Expression of miR-143-3p in SK-MES-1 cells transfected with si-NC or si-PSMG3-AS1 was determined using reverse transcription-quantitative PCR. (D) Luciferase activity was examined in SK-MES-1 cells co-transfected with miR-143-3p mimic, miR-143-3p inhibitor, mimic NC or inhibitor NC, and WT-PSMG3-AS1 or MUT-PSMG3-AS1. ${ }^{* *} \mathrm{P}<0.01$ and ${ }^{* * * *} \mathrm{P}<0.001$ vs. si-NC or mimic NC. ${ }^{\text {\&\&\& }} \mathrm{P}<0.001$ vs. inhibitor NC. PSMG3-AS1, long noncoding RNA PSMG3-antisense 1; si, small interfering; NC, negative control. WT, wild-type; MUT, mutant.

Fig. 4C). Subsequently, a mutated PSMG3-AS1 construct was obtained by mutating the miR-143-3p binding sites within the PSMG3-AS1 3'untranslated region. The luciferase activity of the MUT-PSMG3-AS1-transfected cells was not affected by the expression of miR-143-3p, whereas that of the WT-PSMG3-AS1-transfected cells was significantly decreased 
by $\mathrm{miR}-143-3 p$ overexpression, and increased by miR-143-3p inhibition $(\mathrm{P}<0.001$; Fig. 4D). This suggested that miR-143-3p may bind to the predicted sites and subsequently inhibit the expression of PSMG3-AS1.

\section{Discussion}

The aim of the present study was to investigate the clinical role of PSMG3-AS1, and the interaction between miR-143-3p and PSMG3-AS1, in LUSC. The findings revealed that the expression of miR-143-3p and PSMG3-AS1 was altered in LUSC. Moreover, PSMG3-AS1 may target miR-143-3p to promote cancer cell proliferation.

As noncoding RNAs, IncRNAs and miRNAs are commonly perceived as epigenetic markers for diagnosis and/or prognosis (22-24). However, as a type of IncRNA, PSMG3-AS1 plays a key role in various physiological processes of cancer cells. Therefore, in the present study, the role of PSMG3-AS1 in LUSC was investigated. Firstly, the expression level of PSMG3-AS1 was determined in LUSC tissue samples and four LUSC cell lines. The data demonstrated that PSMG3-AS1 was upregulated in LUSC tissue specimens and all of the cell lines investigated, which suggests that PSMG3-AS1 may serve as an oncogene in LUSC. This is in accordance with PSMG3-AS1 upregulation in glioblastomas and breast cancer cells $(20,25)$. However, a previous study also reported that PSMG3-AS1 was downregulated in pancreatic cancer (23), suggesting that its role may differ between cancer types. Subsequently, further experiments were conducted to verify the role of PSMG3-AS1 in LUSC. PSMG3-AS1 expression, lymph node metastasis and TNM stage were revealed to be associated with the overall survival of patients with LUSC. Additionally, multivariate Cox regression analysis revealed that PSMG3-AS1 expression was an independent prognostic predictor for LUSC. Furthermore, the Kaplan-Meier analysis results confirmed that the high PSMG3-AS1 expression group exhibited a significantly poorer prognosis than the low-expression group in a 5-year follow-up study. Liu et al (23) also identified that PSMG3-AS1 was associated with the 5-year overall survival rate, and was involved in the progression of pancreatic cancer. Yue et al (13) also reported that the increased expression of PSMG3-AS1 in LUAD predicted poor survival. These aforementioned studies indicate that PSMG3-AS1 is associated with poor prognosis in LUSC, in which it may act as a prognostic biomarker.

To investigate the functional role of PSMG3-AS1 in LUSC cell function, loss-of-function experiments were performed by downregulating the expression of PSMG3-AS1 in H520 and SK-MES-1 cells. The results indicated that, compared with untreated cells, si-PSMG3-AS1 inhibited the proliferative, migratory and invasive capacities of LUSC cells. A similar finding was observed in a previous study, whereby transfection with the si-PSMG3-AS1 decreased proliferation in two LUAD cell lines (13). Therefore, we hypothesize that PSMG3-AS1 may serve as an oncogenic factor and may be involved in the progression of LUSC.

PSMG3-AS1 is reported to be upregulated in breast cancer, and may accelerate cancer cell migration and proliferation by sponging miR-143-3p (20). However, PSMG3-AS1 was reported to promote tumor progression by activating the PI3K-Akt pathway $(23,26,27)$. Another study demonstrated that PSMG3-AS1 promoted cancer cell characteristics through the 1ncRNA-PSMG3-AS1/miR-143-3p/COL1A1 signaling axis (20). Furthermore, the relationship between PSMG3-AS1 and miR-143-3p in LUSC was analyzed in the present study. We hypothesized that PSMG3-AS1 may bind miR-143-3p to regulate the biological functions of LUSC cells. In SK-MES-1 cells, PSMG3-AS1-knockdown was demonstrated to upregulate miR-143-3p expression. Simultaneously, Spearman's rank correlation coefficient analysis revealed a negative association between miR-143-3p and PSMG3-AS1 expression. Moreover, the luciferase activity of MUT-PSMG3-AS1-transfected cells was not affected by the expression of miR-143-3p, while that of the WT-PSMG3-AS1-transfected cells was significantly decreased by miR-143-3p overexpression, and increased by miR-143-3p inhibition. Therefore, the present study revealed that PSMG3-AS1 may also drive LUSC cell proliferation, migration and invasiveness by targeting miR-143-3p.

The findings regarding LUSC (namely, an upregulation in PSMG3-AS1) support the recent discussion that PSMG3-AS1 may act as an oncogenic microenvironmental factor $(28,29)$. However, there were limitations to the present study. Firstly, LUSC samples and corresponding normal samples (that were used for determining the expression of PSMG3-AS1) were limited in number. Secondly, in vivo experiments were not performed to verify the effect of PSMG3-AS1 in LUSC.

In conclusion, the results of the current study indicated that PSMG3-AS1 expression was increased in LUSC tissue specimens and cancer cells, and that PSMG3-AS1 overexpression accelerated cancer cell proliferation, migration and invasiveness by targeting miR-143-3p. Moreover, LUSC patients with high PSMG3-AS1 expression exhibited a lower overall survival rate than those with low PSMG3-AS1 expression levels. Therefore, the present study confirms that PSMG3-AS1 has potential as a prognostic biomarker and a future therapeutic target.

\section{Acknowledgements}

Not applicable.

\section{Funding}

The present study was supported by the Liaoning Municipal Basic Medical and Health Technology Project (grant no. S20110167).

\section{Availability of data and materials}

The datasets used and/or analyzed during the current study are available from the corresponding author on reasonable request.

\section{Authors' contributions}

EJ designed the study. $\mathrm{CH}, \mathrm{LZ}, \mathrm{XZ}$ and $\mathrm{ZR}$ conducted the experiments, analyzed the data and revised the manuscript. SC collected the sample tissues and clinical data, and wrote the manuscript. HF interpreted the data. EJ and HF collected the samples and confirm the authenticity of all the raw data. All authors have read and approved the final manuscript. 


\section{Ethics approval and consent to participate}

The present study was approved by the Ethics Committee of The Fourth People's Hospital of Shenyang [approval no. k(2012)11]. Informed consent was obtained from all patients before the initiation of any study-related procedure.

\section{Patient consent for publication}

Not applicable.

\section{Competing interests}

The authors declare that they have no competing interests.

\section{References}

1. Longo DL, Reck M and Rabe KF: Precision diagnosis and treatment for advanced non-small-cell lung cancer. N Engl J Me 377 : 849-861, 2017.

2. Herbst RS, Morgensztern D and Boshoff C: The biology and management of non-small cell lung cancer. Nature 553: 446 2018.

3. Rittmeyer A, Barlesi F, Waterkamp D, Park K, Ciardiello F von Pawel J, Gadgeel SM, Hida T, Kowalski DM, Dols MC, et al: Atezolizumab versus docetaxel in patients with previously treated non-small-cell lung cancer (OAK): A phase 3, open-label, multicentre randomised controlled trial. Lancet 389: 255-265, 2017.

4. Pless M, Stupp R, Ris HB, Stahel RA, Weder W, Thierstein S, Gerard MA, Xyrafas A, Früh M, Cathomas R, et al: Induction chemoradiation in stage IIIA/N2 non-small-cell lung cancer: A phase 3 randomised trial. Lancet 1049-1056, 2015.

5. Song Y, Zhou B, Du X, Wang Y, Zhang J, Ai Y, Xia Z and Zhao G: Folic acid (FA)-conjugated mesoporous silica nanoparticles combined with MRP-1 siRNA improves the suppressive effects of myricetin on non-small cell lung cancer (NSCLC). Biomed Pharmacother 125: 109561, 2020.

6. Gandara DR, Hammerman PS, Sos ML, Lara PN Jr and Hirsch FR: Squamous cell lung cancer: From tumor genomics to cancer therapeutics. Clin Cancer Res 21: 2236-2243, 2015.

7. Mentzelopoulos A, Gkiatis K, Karanasiou I, Karavasilis E, Papathanasiou M, Efstathopoulos E, Kelekis N, Kouloulias V and Matsopoulos GK: Chemotherapy-induced brain effects in small-cell lung cancer patients: A multimodal MRI study. Brain Topogr 34: 167-181, 2021.

8. Chen Z, Fillmore CM, Hammerman PS, Kim CF and Wong KK: Non-small-cell lung cancers: A heterogeneous set of diseases. Nat Rev Cancer 14: 535-546, 2014.

9. Lo YL, Hsiao CF, Chang GC, Tsai YH, Huang MS, Su WC, Chen YM, Hsin CW, Chang CH, Yang PC, et al: Risk factors for primary lung cancer among never smokers by gender in a matched case-control study. Cancer Causes Control 24: 567-576, 2013.

10. Kabir Z, Bennett $\mathrm{K}$ and Clancy L: Lung cancer and urban air-pollution in Dublin: A temporal association? Ir Med J 100: 367-369, 2007

11. Loomis D, Grosse Y, Lauby-Secretan B, El Ghissassi F, Bouvard V, Benbrahim-Tallaa L, Guha N, Baan R, Mattock H, Straif $\mathrm{K}$, et al: The carcinogenicity of outdoor air pollution. Lancet Oncology 14: 1262-1263, 2013.
12. Torre LA, Bray F, Siegel RL, Ferlay J, Lortet-Tieulent J and Jemal A: Global cancer statistics, 2012. CA Cancer J Clin 65: 87-108, 2015.

13. Yue N, Ye M, Zhang R and Guo Y: miR-449b-5p targets lncRNA PSMG3-AS1 to suppress cancer cell proliferation in lung adenocarcinoma. BMC Pulm Med 20: 152, 2020.

14. Gao Y, Meng H, Liu S, Hu J, Zhang Y, Jiao T, Liu Y, Ou J, Wang D, Yao L, et al: lncRNA-HOST2 regulates cell biological behaviors in epithelial ovarian cancer through a mechanism involving microRNA let-7b. Hum Mol Genet 24: 841-852, 2015.

15. Katsushima K, Natsume A, Ohka F, Shinjo K, Hatanaka A, Ichimura N, Sato S, Takahashi S, Kimura H, Totoki Y, et al: Targeting the Notch-regulated non-coding RNA TUG1 for glioma treatment. Nat Commun 7: 13616, 2016.

16. Schaukowitch K and Kim TK: Emerging epigenetic mechanisms of long non-coding RNAs. Neuroscience 264: 25-38, 2014.

17. Monroig Pdel C, Chen L, Zhang S and Calin GA: Small molecule compounds targeting miRNAs for cancer therapy. Adv Drug Deliv Rev 81: 104-116, 2015.

18. Cheng G, Liu D, Liang H, Yang H, Chen K and Zhang X: A cluster of long non-coding RNAs exhibit diagnostic and prognostic values in renal cell carcinoma. Aging (Albany NY) 11: 9597-9615, 2019.

19. Simpson RJ, Lim JW, Moritz RL and Mathivanan S: Exosomes: Proteomic insights and diagnostic potential. Expert Rev Proteomics 6: 267-283, 2009.

20. Cui Y, Fan Y, Zhao G, Zhang Q, Bao Y, Cui Y, Ye Z, Chen G, Piao X, Guo F, et al: Novel lncRNA PSMG3AS1 functions as a miR 1433p sponge to increase the proliferation and migration of breast cancer cells. Oncol Rep 43: 229-239, 2020.

21. Livak KJ and Schmittgen TD: Analysis of relative gene expression data using real-time quantitative PCR and the 2(-Delta Delta C(T)) method. Methods 25: 402-408, 2001.

22. Jiang $W$ and Wang $M$ : New insights into the immunomodulatory role of exosomes in cardiovascular disease. Rev Cardiovasc Med 20: 153-160, 2019.

23. Liu Y, Huo Z, Wang W, Zhai S, Wang Y, Weng Y, Deng X and $\mathrm{Lu} \mathrm{X}$ : Construction and integrated analysis of a lncRNA-associated competing endogenous RNA network reveal functional lncRNAs in pancreatic cancer. Transl Cancer Res 9: 3643-3657, 2020.

24. Liu QY, Miao Y, Wang XH, Wang P, Cheng ZC and Qian TM: Increased levels of miR-3099 induced by peripheral nerve injury promote Schwann cell proliferation and migration. Neural Regen Res 14: 525-531, 2019.

25. Chen L, Wang G, Xu Z, Lin K, Mu S, Pan Y and Shan M Overexpression of 1ncRNA PSMG3-AS1 distinguishes glioblastomas from sarcoidosis. J Mol Neurosci 70: 2015-2019, 2020.

26. Okkenhaug $\mathrm{K}$ and Vanhaesebroeck B: PI3K in lymphocyte development, differentiation and activation. Nat Rev Immunol 3: 317-330, 2003.

27. Meo AD, Bartlett J, Cheng Y, Pasic MD and Yousef GM: Liquid biopsy: A step forward towards precision medicine in urologic malignancies. Mol Cancer 16: 80, 2017.

28. Kalwa M, Hänzelmann S, Otto S, Kuo CC, Franzen J, Joussen S, Fernandez-Rebollo E, Rath B, Koch C, Hofmann A, et al: The lncRNA HOTAIR impacts on mesenchymal stem cells via triple helix formation. Nucleic Acids Res 44: 10631-10643, 2016.

29. Liu Z, Chen Z, Fan R, Jiang B, Chen X, Chen Q, Nie F, Lu K and Sun M: Over-expressed long noncoding RNA HOXA11-AS promotes cell cycle progression and metastasis in gastric cancer. Mol Cancer 16: 82, 2017. 